\title{
Artificial Intelligence Three Dimensional Solar Tracker
}

\author{
Ashardi Abas ${ }^{1}$
}

\begin{abstract}
Renewable energy is playing an importance role as fuel price energy prices unstable. Solar energy is one of the furthermost popular renewable energy sources. This research illustrated the design and development of artificial Intelligence three dimensional microcontroller tracking system. By using this method is able to control the panel for optimizing and maximizing the amount of solar energy that can be received from the sun by controlling the rotation of the panel

It also demonstrated the design and construction of the system. PIC16F877 Microcontroller is used to control the three dimensional tracking systems. This paper also covers the design and construction of the Solar Tracking mechanical system with the other peripheral electronic circuits. One stepper motors is attach to control the altitude angle, and one stepper Motor for the elevation angle. Four pairs of Light Dependent Resistor sensors (LDR) were used for distinguished the proportion of light intensity. The PIC16f877 is program using assembly language in Assemble for PIC. A real model of the system was demonstrated to validate the design. The system outlined in this research proved to be successful in detecting the direction of the sun movement.
\end{abstract}

Index Terms - Solar Energy, Solar Panel, Microcontroller, Stepper Motor.

\section{INTRODUCTION}

Improving the converting of solar energy to electric supply is an attention to the future energy. Solar panel are usually installed in domestic and commercial roof fittings without a sun tracking structures and use basic fitting to enhance solar energy collection. A bigger scale solar energy collection plants also used massive and expensive standard fixing by just concentrate to a direct sunlight $[1,2]$. But, most of these systems did not take an advantage of the intelligence self-adjusted three-dimensional, normally the solar energy collection mostly happens on flat surface in contrast with what is generally observed [3,4]. This report show a formulate, by solving it using an intelligence system and proving this study via experiment and solve the problem of collecting solar energy in three-dimensions [5].

Commonly there are three different approaches of increasing the collection of solar energy. These include a fix focusing toward the solar panel, following the path of the sun using fixed control algorithms, and intelligence dynamic tracking. The first method involves focusing incident rays onto a solar panel; this allows incident rays to reach the array normal to the panel surface. The second method uses a device controller to determine the position of the sun with reference to the previous history of current day, month and year. The dynamic tracking is similar to this method; however sensors are used to

Ashardi Abas ${ }^{1}$ Computing Department, FSKIK, UPSI Malaysia. determine the current position of the sun light source. The dynamic tracking system was chosen because it proposed the most accurate method of maintaining optimum power collection possible.

\section{SYSTEM OVERVIEW}

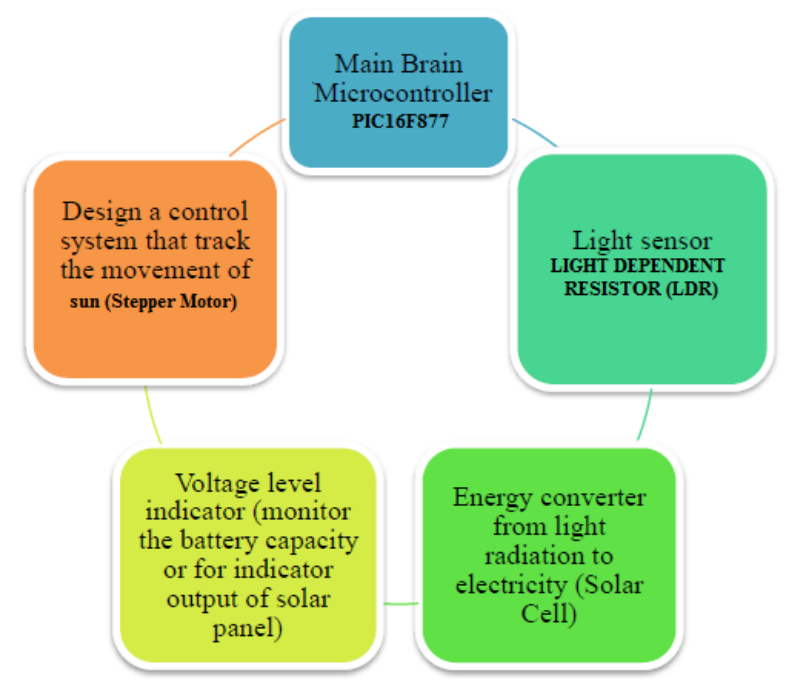

Fig1. Scope of Research.

The main aim of the research is to demonstrate intelligence solar tracking system methods that involve both hardware and software development. To achieve this result a microcontroller of Pic16f877 were used, four LDR were setup that capable to distinguish the light intensity and a set of stepper motor to control the rotation of the solar panel refer figure 1 . We implement this technique using an intelligence programming by using an assembly languages and loading the code program into microcontroller through ISP (in system programmer). The current state data will go through a built in ADC inside the PIC16f877 and later on the stepper motor were rotate according to the specific algorithm that being programmed. The stepper motor is not directly connected to microcontroller due to safety reason. The output of the solar panel will be monitor by the Voltage Level Indicator and also be used for monitoring the battery voltage level.

\section{LITERATURE REVIEW}

\section{A. Maximize power.}

Some literature review was conducted and fundamental steps being investigated. The most important part is to examine the suitable current tracking methods from the solar panel. By doing this it can maximize the power obtained from the solar panel. 
Through experiments conducted during research by [6] it was concluded that the current obtained from solar cells is influenced by the angle at which incident rays strike the cell surface. By using a stationary light source and adjusting the angle at which the light rays strike the cell, a plot of current delivered vs. angle of incidence can be created. This property of solar cells is confirmed by the data contained in Table 1 and illustrated by Figure 2 .

TABLE 1.1

CURRENT DELIVERED For VARIOUS ANGLES OF INCIDENCE

\begin{tabular}{|c|c|}
\hline $\begin{array}{l}\text { ANGLE OF } \\
\text { INCIDENCE } \\
\text { IN DEGREES }\end{array}$ & $\begin{array}{l}\text { CURRENT } \\
\text { DELIVERED } \\
\text { IN mAs }\end{array}$ \\
\hline 0 & 5 \\
\hline 5 & 5 \\
\hline 10 & 5 \\
\hline 15 & 5 \\
\hline 20 & 5 \\
\hline 25 & 4 \\
\hline 30 & 4 \\
\hline 35 & 4 \\
\hline 40 & 4 \\
\hline 45 & 3 \\
\hline 50 & 3 \\
\hline 55 & 3 \\
\hline 60 & 2 \\
\hline
\end{tabular}

From the experimental data investigation, to maintain the optimum power output from a solar panel, the angle of incidence must be at zero degrees $\left(0^{\circ}\right)$. Therefore the panel needs constantly facing toward the sun light. This involves a tracking system that can constantly align the panel toward the preferred position.

\section{CURRENT DELIVERED vs. ANGLE OF INCIDENCE}

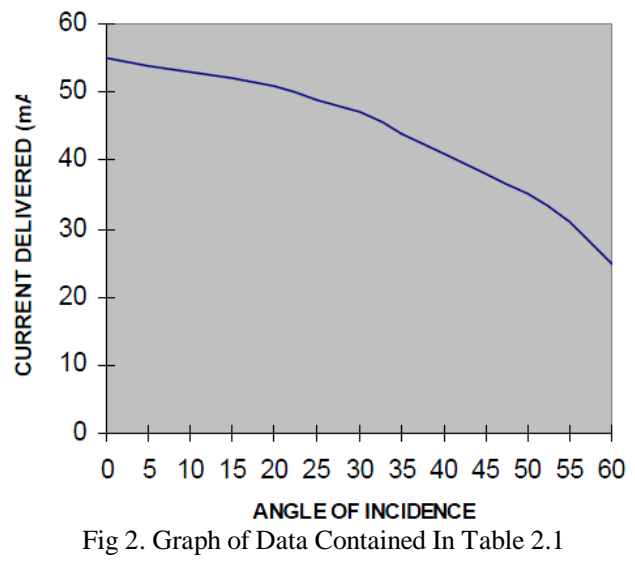

\section{B. Tracking Techniques}

There are numerous methods of tracking presently presented; these differ mainly in the method of applying the designs. The two common methods of tracking used are dynamic tracking and fixed control algorithms.

The essential variance between the two methods is the way in which the sun light path is determined. The dynamic tracking system actively searches for the sun's current position at any time of day (or night). For the fixed control algorithm systems on the other hand, the path of the sun is determined by refer to an algorithm that calculates the position of the sun from the precious history. So the control system did not dynamically find the sun's position but referring out to specific current time, day, month, and year.

The common things for both methods of tracking it have a control system. The system comprises of some method of direction control, such as DC motors, servo motors and stepper motors, which are control via a controller circuit.

\section{SYSTEM DEVELOPMENT}

The rotation of the solar panel mechanical systems would need two separate of stepper motors, which capable to control the position of the panel in three dimensional positions. The control circuit needs to be adding to control the movement the motors.

\section{A. Motors Selection}

For the accurate result for this type of application stepper motor were being used. Stepper motors were used due to high torque and it precision. Firstly, they cannot run freely by themselves. Stepper motors do as their name suggests they "step" a little bit at a time. Stepper motors also different from DC motors in their torque-speed relationship. DC motors generally are not very good at producing high torque at low speeds, without the aid of a gearing mechanism. Stepper motors, on the other hand, work in the opposite manner. They produce the highest torque at low speeds. Stepper motors also have another characteristic, holding torque, which is not present in DC motors. Holding torque allows a stepper motor to hold its position firmly when not turning. This can be useful for applications where the motor may be starting and stopping, while the force acting against the motor remains present. This eliminates the need for a mechanical brake mechanism. Steppers don't simply respond to a clock signal, they have several windings which need to be energized in the correct sequence before the motor's shaft will rotate. Reversing the order of the sequence will cause the motor to rotate the other way. If the control signals are not sent in the correct order, the motor will not turn properly. It may simply buzzing sound and not move, or it may actually turn, but in a rough or jerky manner. A circuit which is responsible for converting step and direction signals, patterns is called a translator.

\section{B. Motors Drivers}

In order to make sure the stepper motor running smoothly, its need a driver or translator to generate a specific sequence as an input data to the stepper motor. The drive circuitry chosen for the unipolar stepper motors is simple Darlington transistor circuits. The UCN5804 chip was used for the drive circuit as it incorporates 4 Darlington transistors, since four transistors are needed for each of the stepper motors. By using this method driving stepper motor is more accurate and efficiency refers Figure 3. 

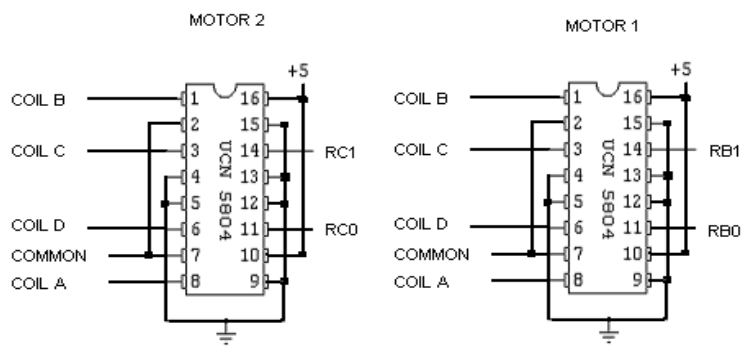

Fig.3. Driver IC for Stepper Motor 1 and 2

Combining with a low-power CMOS logic with high-current and high-voltage bipolar outputs, the UCN5804 drivers provide complete control and drive for a four-phase unipolar stepper motor with continuous output current ratings to $1.25 \mathrm{~A}$ per phase (1.5 A startup).

\section{Sensor Component}

After some research being made a light dependent resistor (LDR) was chose in order to distinguish the light intensity. The light-sensitive part of the LDR is a wavy track of cadmium sulfide. Light energy triggers the release of extra charge carriers in this material, so that its resistance falls as the level of illumination increases. Therefore the brighter the light will make resistance become lower. The resistance of the sensor varies based on the amount of light that hits it. The resistance can vary from $300 \mathrm{~K}$ in the dark to $1 \mathrm{~K}$ in the light. The observation of light intensity attenuation has to be experimentally verified. The design only wants to seek the variation of light when the intensity is changed by referring to figure 4 some calculations have to be verified. It can be proved by using the simple Ohm's Law: V = IR. By series the circuit the voltage drop across each network resistor will be divided regarding voltage divider rules.

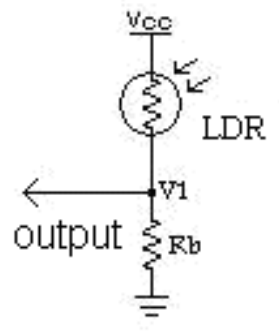

Fig 4. Voltage Divider Concepts

To convert the resistance into a voltage, we use a second resistor $\mathrm{Rb}$. Then, assuming that no current goes into pin output, you can find V1. To find V1 you can use Ohms Law. With $\mathrm{Vcc}=5$, Ohms Law gives you (5 - V1) $/ \mathrm{R}_{\mathrm{LDR}}=\mathrm{V} 1 / \mathrm{Rb}$ where $R_{L D R}$ is the resistance of the sensor (LDR). Then you can solve for $\mathrm{V} 1=5 \times \mathrm{Rb} /\left(\mathrm{R}_{\mathrm{LDR}}+\mathrm{Rb}\right)$

The maximum voltage is when $R_{L D R}$ is at its minimum $(1.37 \mathrm{~K} \Omega)$, Then the minimum voltage is when $\mathrm{R}_{\mathrm{LDR}}$ at its maximum $(116 \mathrm{~K} \Omega)$.

We set the maximum voltage is around $4.8 \mathrm{~V}$.

$\mathrm{V}_{\max }=\mathbf{4 . 8 \mathrm { V }}$

The equation $4.8 \mathrm{~V}=5 \mathrm{~V} \mathrm{x} \mathrm{Rb} /\left(\mathrm{R}_{\mathrm{LDR}}+\mathrm{Rb}\right)$

$4.8 \mathrm{~V}=5 \mathrm{~V} \mathrm{x} \mathrm{Rb} / 1.37 \mathrm{~K} \Omega+\mathrm{Rb}$

$4.8 \mathrm{~V}(1.37 \mathrm{~K} \Omega+\mathrm{Rb})=5 \mathrm{~V} \times \mathrm{Rb}$

$$
\begin{aligned}
& 6576+4.8 \mathrm{Rb}=5 \mathrm{~V} \times \mathrm{Rb} \\
& (6576+4.8 \mathrm{Rb}) / 5 \mathrm{v}=\mathrm{Rb} \\
& 1315.6+0.96 \mathrm{Rb}=\mathrm{Rb} \\
& 1.96 \mathrm{Rb}=1315.6 \\
& \mathrm{Rb}=1315.6 / 1.96 \\
& \mathrm{Rb}=\mathbf{6 7 1 . 2 2} \boldsymbol{\Omega}
\end{aligned}
$$

We already know the value of $\mathrm{Rb}$ so we find the value of minimum voltage. By using the same formula, but change the value of $\mathrm{R}_{\mathrm{LDR}}(116 \mathrm{~K} \Omega)$.

The equation $\mathrm{V}_{\min }=5 \mathrm{~V} \times 671.22 \Omega /(116 \mathrm{~K} \Omega+671.22 \Omega)$

$$
\mathrm{V}_{\min }=\mathbf{0 . 0 2} \mathrm{V}
$$

Using the equations for $\mathrm{V} 1$ max and min we can determine that $670 \Omega$ is a good value for Rb. $670 \Omega$ gives you a wide voltage range from minimum to maximum. Now we have a sensor voltage. V1, that varies from about 0.1 volts to 4.8 volts. The LDR will be an output voltage attenuate from 0 Volt to 5 Volt regarding from the intensity of the light.

\section{Sensor position}

To design the sensor circuit, a suitable method for determining the position of the sun was needed. This involved a process of design and testing to establish the most efficient and accurate method. After testing several designs, the most effective design was found to be a simple four-sided LDR.

The design incorporates four LDR acting as sensors. Each sensor is positioned on the top of the panel so that it the sensor will receive a maximum amount of voltage reading. This is shown in Figure 5.
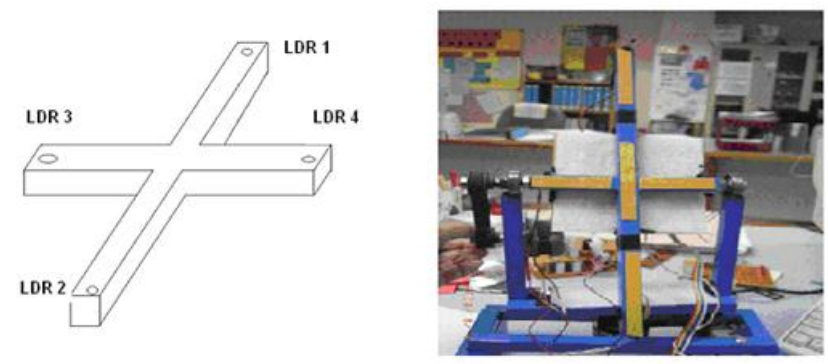

Fig 5. Sensor Position

The sensors are arranged so that the voltage across (LDR1 \& LDR2 for tilt, LDR3 \& LDR4 for rotation) will be able the get equal values when the system at the maximum intensity from the sun. The controller will fetch the value of sensors from tilt section and comparing them digitally, if they are not equal the motor 2 that attach to tilt section will move toward the highest sensor reading. It only stops when two of the sensors are equal.

To simplify the comparison even more, two opposing sensors are dedicated to rotating and two are dedicated to tilting. Due to the fact that the controller reads the voltage output from the sensors, it is necessary to set the operating range of the sensors to an appropriate voltage range. The sensor reading will be 0 Volt when there is no intensity (no light sources) and it will increase depends on the intensity reading value. The intensity is proportional to the voltage value referring figure 6 . 
(v)

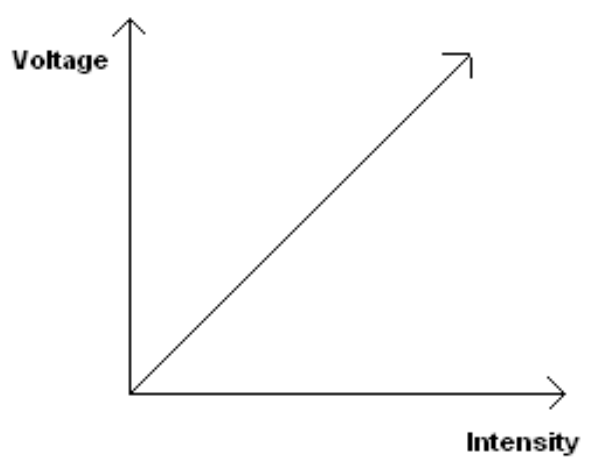

Fig 6. Voltages Against Intensity of Light

The voltage output is in the form of the analogue signal, in order to make a simple circuit; we need to investigate to use a controller that has a capability to read an analogue signal.

\section{A. Micro-Controller component selection}

When choosing a micro-controller chip for the tracking system, it was important to consider the functions that need to perform. The functions include converting the analog voltages from the sensor circuits into digital values that can then to be compared. The micro-controller also needs the capacity to handle inputs from the user interface and the outputs of the stepper motor control circuit. These inputs and outputs need to be clarified before the micro-controller is chosen.

To handle the analog to digital (A/D) conversions, the control chip must read various voltage levels simultaneously and continuously. The user interface requires one switch to select between automatic and manual tracking and four pushbuttons to control direction when using manual control. The output to the motor circuit requires four data channels to control movement of the stepper motors (two each).

After researching an appropriate control chip, the PIC16F877 micro-controller was found to be the preferred choice as it can perform all required functions using only a single chip. The chip contains an on chip A/D converter, adequate programmable memory space, ample input and output pins and a supply voltage of five volts and have 5 different ports (Port A, B, C, D and E).

This project consists of a micro-controller of PIC 16F877:

- Micro-Controller 1 (PIC 16f877) to control 2 stepper motor and read 4 IR sensor analogue signals.

The micro-controller required controlling 2 motors referring from 4 IR input sensor to position the panel according to the array of reading input. Each stepper motor required five data channels (inputs) for rotation to be controlled.

Taking into consideration all of the necessary design specifications, the PIC 16F877 microprocessor was chosen as the control chip (figure 7). This was a reasonable choice for this application as the device contains:

- An 8 channel built 10 bit A/D converter

- $2 \mathrm{~K}$ bytes of EEPROM

- $\quad$ Five 8-bit I/O ports

The PIC acts as the brains and control the robots based on inputs from the various sensors. The PIC contains 356 bytes of RAM and $8 \mathrm{~K}$ of flash ROM. Two PWM pins allow for easy output waveform generation and built in $\mathrm{A} / \mathrm{D}$ pins allow for sensor input.

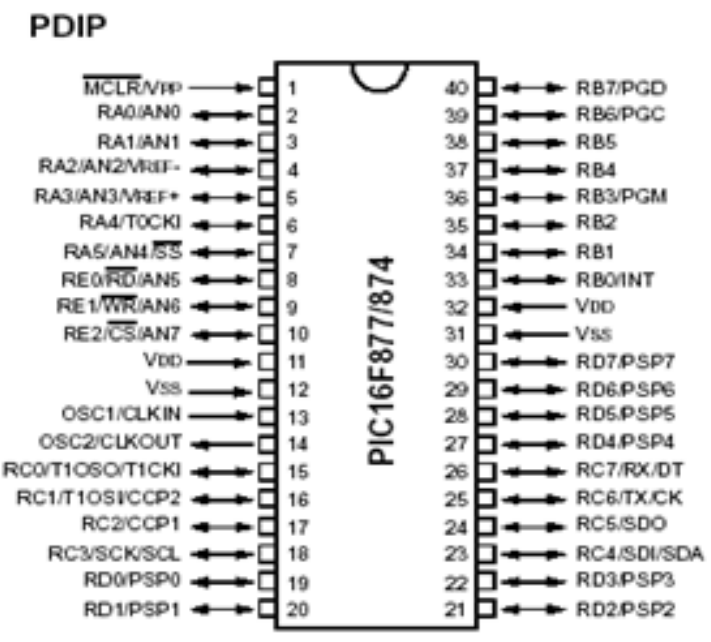

Fig 7. PIC 16F877 Pin Configuration.

\section{Operation CONTROL}

The processor controls the stepper motors by setting pins on the output ports that are connected to the IC driver or the translator motor drive circuit. These pins represent the windings of the motors and by outputting the appropriate sequence the motors can be steeped in either direction. It only used 2 output ports for each stepper motor RB0 \& RB1 for stepper motor tilt operation and $\mathrm{RC} 0$ \& $\mathrm{RC} 1$ for rotating stepper motor.

\section{A. Code program}

The program has been written in assembler language code for the PIC 16F877 microprocessor. The program can run in automatic mode. In automatic mode the voltages are fed by the sensor are directly transferred to the A/D register. From the $\mathrm{A} / \mathrm{D}$ register the processor directly comparing them digitally by using general purpose registers as the medium of comparing technique. After the process is completed, the processor uses a simple algorithm to move the stepper motors in either or vice versa direction. A flowchart of the Program is given in Figure 8. 


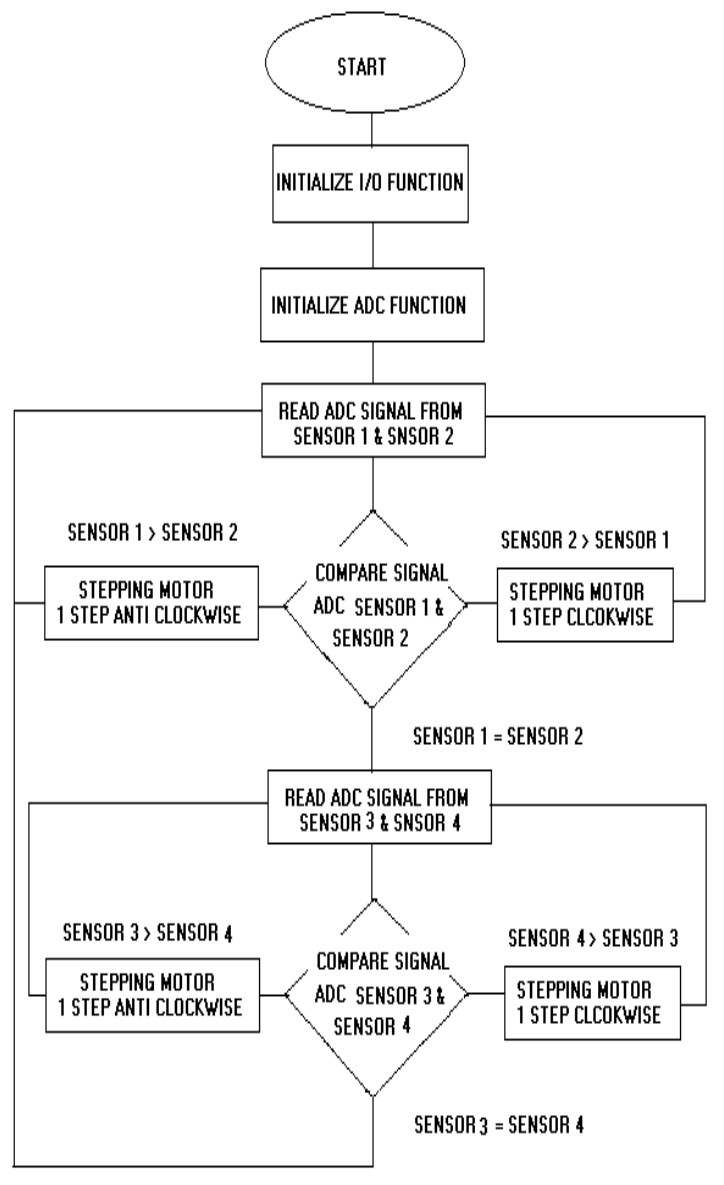

Fig 8. Software Flowchart

\section{B. Supporting software}

The development of code for the programming section needs several supporting software starting from the compiler until downloading code to the micro controller.

i. MPASM is the notepad to create the raw code and being used as a compiler to detect any error that occurs during the code development. It also will convert the assembly code the execution if there is no error occurs.

ii. IC Prog is the software that used to transfer the execution code to the micro controller integrated circuit.

iii. JDM programmer is the intermediate device to transfer the code to the controller IC by using rs232 technique.

\section{DISCUSSION}

Upon examination of the results obtained from the tests with the stationary array and those obtained from the sun-tracking array, it was obvious that the sun-tracking method is more effective in gathering solar energy refer figure 9. This was the expected outcome of the research, with the current system meeting all of the required goals set out. The system itself is a very effective solution to the initial problem posed. Although it is not the only solution available, it is however, the most efficient given the time, budget and material restrictions.

\section{Sun-Tracking System vs Stationary System}

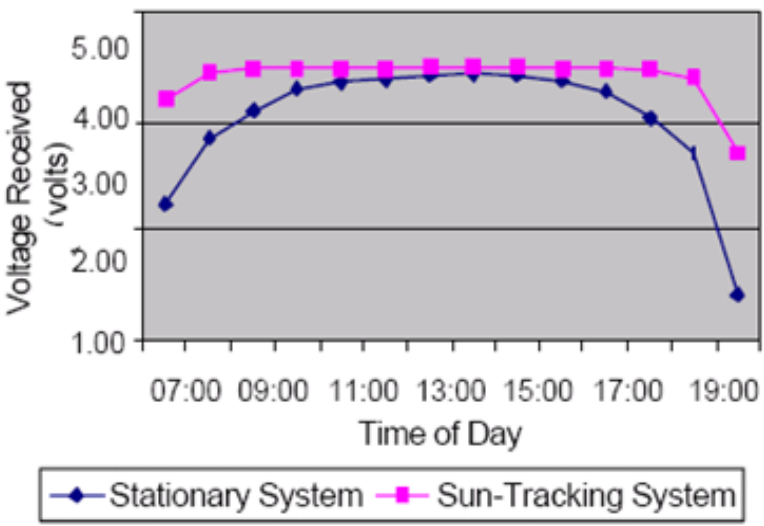

Fig 9. Result From Experiment

As can be seen from the above graph, the sun tracking system collects the maximum amount of solar energy as possible across the entire day, whereas, the stationary system only collects maximum energy when the sun is overhead. From these results it can be seen that the stationary system is inefficient in receiving solar energy in the early and late stages of the day.

\section{CONCLUSION}

In terms of the system mechanism able to get same coordinated, although several alignments and movement have been made. But if we consider from the standard deviation of the position, it might be around $99.5 \%$ of all measurements fall. This figure will vary over the workspace, especially near the boundaries of the workspace.

The payload of the system indicates that it's able to do the job excellently by maneuvering rotating and tilting perfectly. It is possible to exceed the maximum payload, and still have the system operate.. When the system is accelerating fast, the payload should be less than the maximum mass. This is affected by the ability to firmly grip the part, as well as the system structure, and the actuators.

The maximum intensity of light position is successfully being tracked. Both motors work well to rotate the panel system, based on the sensory input as being planned.

Although the motor work with $5 \mathrm{~V}$ voltage source the accuracy to turn according to 1 single step have been achieved. The motor managed to maneuver if any changes happen to sensory input. The controller reacts 100\% excellent according to the software programming. It managed to sample the analogue input from the sensor and convert it to 8-bit ADC resolution. But in future implementation it should be upgraded to distinguish to respond to 10-bit $\mathrm{ADC}$ resolution system, in order to get a more accurate result.

System comparing input ADC from various input ADC channels from PIC16F877 is very flexible were the respond in real time application. The gearing system design should be improved in order to cut down the angle into the much smaller angle of degree. The stepper motor should be changed if wants to carry more load in future expansions. 


\section{REFERENCES}

[1] S. Chen, B. Mulgrew, and P. M. Grant, "A clustering technique for digital Myers, B., Bernardi, M. \& Grossman, J. C. Three-dimensional photovoltaics. Appl.Phys. Lett. 96, 071902 (2010).

[2] Meinel, A. B. \& Meinel, M. P. Applied Solar Energy, page (Addison Wesley, 1976).

[3] Reda, I. \& Andreas, A. Solar position algorithm for solar radiation applications. Solar Energy 76, 577 (2004).

http://dx.doi.org/10.1016/j.solener.2003.12.003

[4] Lave, M. \& Kleissl, J. Solar variability over various timescales using the top hat wavelet. American Solar Energy Society Conference (2011). In press.

[5] Shonkwiler, R. W. \& Mendivil, F. Explorations in Monte Carlo Methods, 145 (Springer, 2009). http://dx.doi.org/10.1007/978-0-387-87837-9

[6] Elliot Larard (1998), Research of Sun Tracking Solar Array System, University of Queensland, pp. 5.

[7] Microchip Technology Inc, "HI-TECH C Compiler for PIC10/12/18 MCUs Version 12.80 Release Notes

[8] Microchip Technology Inc, "Software Solutions for the 16-bit and 32-bit Designer," January 2015

[9] "PIC18F8722 Family Data Sheet," Microchip

[10] Dogan Ibrahim, "Microcontroller DEBUGGING and TESTING tools," Electronics World, August 2013, pp. 20-23

[11] "MPLAB ICD 3 In-Circuit Debugger User"s Guide, " Microchip

[12] PIC 16F877A Manual Datasheet, Microchip Technology Inc. www.microchip.com

[13] Lawrence A. Duarte. The Microcontroller Beginner's Handbook. 2 ndEdition. United States of America: Prompt Publication. 3-5;2013.

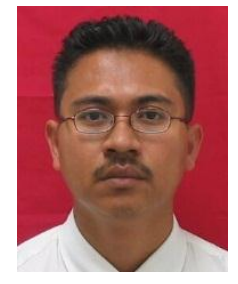

Mr. A.Abas was born in Kuantan Pahang, Malaysia on $24^{\text {th }}$ May 1976. Education background: Phd. Artificial Intelligence, Bradford University UK(Jan 2006-Nov 2010), M.Sc Mechatronic Eng specialized in Signal \& system Faculty of Mechtronic Engineering, IIUM, Malaysia (November 2000 - august 2002) and B.Eng (Hons) Electrical \& Electronic Engineering, University of Hertforshire, UK (Sept 98 - June 1999), Teaching Certificate level IV by City \& Guild london (Dec 97- June 98), Higher National diploma in Electrical \& Electronic Engineering edexcel UK (August 94 - May 1997).

Working Experience: (July 2005 - Present) LECTURER, Faculty of Art, Computing and Industry Creative Universiti Pendidikan Sultan Idris, Tanjong Malim, Perak,Malaysia (nov 2012 - nov 2014) Post Graduate Researcher General Research Liverpool John Moor University, (July 99 - June 2005) Lecturer UniKL BMI Malaysia, (June 1998 - Sept 1998), Trainer at British Aerospace Stockport,Manchester UK (RJ45 manufacturing), (May 1997-Dec1997) Assistance Engineer Pernec Corp ampang. Email: ababas@bradford.ac.uk. 